\title{
Nonlinear propagation of broadband intense electromagnetic waves in an electron-positron plasma
}

\author{
M. Marklund, ${ }^{1,2}$ B. Eliasson ${ }^{3}$ and P. K. Shukla ${ }^{3,1,4,5,6}$ \\ ${ }^{1}$ Centre for Nonlinear Physics, Department of Physics, \\ Umeå University, SE-90187 Umeå, Sweden \\ ${ }^{2}$ Centre for Fundamental Physics, Rutherford Appleton Laboratory, \\ Chilton, Didcot, Oxon OX11 OQX, U.K. \\ ${ }^{3}$ Institut für Theoretische Physik IV and Centre for Plasma Science and Astrophysics, \\ Fakultät für Physik und Astronomie, \\ Ruhr-Universität Bochum, D-44780 Bochum, Germany \\ ${ }^{4}$ CCLRC Centre for Fundamental Physics, \\ Rutherford Appleton Laboratory, Chilton, Didcot, Oxon OX11 OQX, U.K. \\ ${ }^{5}$ SUPA, Department of Physics, University of Strathclyde, Glasgow G4 ONG, U. K. \\ ${ }^{6}$ GoLP/Centro de Física de Plasmas, \\ Instituto Superior Técnico, 1096 Lisboa Codex, Portugal
}

\begin{abstract}
A kinetic equation describing the nonlinear evolution of intense electromagnetic pulses in electron-positron (e-p) plasmas is presented. The modulational instability is analyzed for a relativistically intense partially coherent pulse, and it is found that the modulational instability is inhibited by the spectral pulse broadening. A numerical study for the one-dimensional kinetic photon equation is presented. Computer simulations reveal a Fermi-Pasta-Ulam-like recurrence phenomena for localized broadband pulses. The results should be of importance in understanding the nonlinear propagation of broadband intense electromagnetic pulses in e-p plasmas in laserplasma systems as well as in astrophysical plasma settings.
\end{abstract}

PACS numbers: 52.38.-r, 52.27.Ny, 52.65.Rr 


\section{INTRODUCTION}

Electron-positron plasmas are believed to be an important ingredient of the early universe and in astrophysical objects such as pulsars, supernova remnants and active galactic nuclei, and in gamma-ray bursts [1]. In such extreme environments, the electron-positron pairs may be created by collisions between particles that are accelerated by electromagnetic and electrostatic waves and/or by gravitational forces. In pulsar environments, there is also a possibility of pair creation via high-energy curvature radiation photons that are triggered by charged particles streaming along the curved magnetic field [2], with a resulting collection of positrons at the polar caps of the pulsar [3, 4]. High-energy laser-plasma interactions and fusion devices on Earth also constitute a source of electron-positron plasmas. Experiments with petawatt lasers (with intensities exceeding $10^{20} \mathrm{~W} / \mathrm{cm}^{2}$ ) have demonstrated the production of $\mathrm{MeV}$ electrons and evidence of positron production via electron collisions 5, 6]. Positrons are also believed to be created in post-disruption plasmas in large tokamaks through collisions between $\mathrm{MeV}$ electrons and thermal particles [7].

Various collective phenomena in electron-positron plasmas have been demonstrated in the laboratory, such as the creation of wake fields by ultra-relativistic positron beams [8] and two-stream instabilities in streaming electron-positron plasmas [9]. The natural saturation mechanism for a two-stream instability is particle trapping in which electrons and positrons are trapped in the electrostatic potential of the large-amplitude wave. Theoretical and numerical studies have shown that solitary electron/positron holes can exist both in a nonrelativistic pair plasma [10, 11, 12] and in a relativistically hot electron-positron-ion plasma 13]. Intense electromagnetic radiation introduces a new nonlinearity in the electron-positron plasma via the relativistic mass increase of the plasma particles in the quivering electric field of the electromagnetic waves [14, 15]. It has been demonstrated theoretically that relativistic electromagnetic solitary waves can exist in a cold electron-positron plasma, if there is an inclusion of massive ions [18]. Several authors [16, 17] have also considered the nonlinear propagation of small amplitude electromagnetic waves and Alfvén vortices in a strongly magnetized electron-positron plasma.

Over recent years, the laser pulse intensity has been strongly increasing, and over the next five to ten years it is expected to reach focal intensities of $\sim 10^{26} \mathrm{~W} / \mathrm{cm}^{2}$ [19]. Thus, the interaction between plasmas and intense laser pulses is currently of great interest [20, 
21, 22, 23, 24]. As laser intensities keep increasing, laboratory plasma experiments will experience the effects of the nonlinear quantum vacuum [25], such as pair creation and photon splitting. Theoretical studies of nonlinear laser-plasma interactions mostly make the simplified assumption of a coherent, monochromatic wave propagation in plasmas. It is then relatively easy to derive analytic expressions for various instabilities (e.g. relativistic Raman forward and backward scattering instabilities and the modulational instability in an electron-ion plasma [26]) and the formation of envelope electromagnetic solitons [27].

However, the assumption about a monochromatic wave is not always true, e.g. if the electromagnetic waves are excited via turbulent processes. Then, we would have a distribution of waves with different wavelengths and frequencies that are only partially coherent. Theories for photon acceleration of partially coherent waves have been developed in the framework of nonlinear optics [28] and are based on the Wigner's kinetic description [29]. The latter has been used to investigate the nonlinear instability of random phased small amplitude electromagnetic waves in a nonlinear dispersive medium [30].

In this paper, we consider the nonlinear propagation of relativistically intense broadband laser pulses in an electron-positron plasma. In order to analyze the properties of such partially coherent laser pulses, we perform a Wigner analysis of the modified nonlinear Schrödinger equation, and obtain a wave kinetic equation for the photon quasi-particles. The resulting kinetic equation is investigated both analytically and numerically. Specifically, we present the modulational instability and the dynamics of partially coherent intense laser pulses. It is found that the partial coherence of the laser pulse yields a reduced growth rate for the modulational instability. The fully nonlinear evolution of the wave kinetic equation is investigated by means of numerical simulations. The latter show a complex dynamics of broadband laser pulses in that the laser envelope contracts and disperses in a quasi-periodic manner, somewhat similar to the Fermi-Pasta-Ulam recurrence phenomenon encountered for the nonlinear Schrödinger equation. The present results should be useful for understanding the nonlinear propagation of broadband intense electromagnetic pulses through pair plasmas such as those in laser-plasma systems and in astrophysical settings. 


\section{GOVERNING EQUATIONS AND THE MODULATIONAL INSTABILITY}

Let us consider the nonlinear propagation of intense circularly polarized electromagnetic waves in pair plasmas without ions. Accounting for the relativistic mass increase of the pairs and quasi-stationary density fluctuations driven by the relativistic ponderomotive force, Shukla et al. [31] derived the fully nonlinear equation

$$
i \frac{\partial a}{\partial t}+\frac{1}{2} \nabla^{2} a+\left[1-\frac{\exp \left[\beta\left(1-\sqrt{1+|a|^{2}}\right)\right]}{\sqrt{1+|a|^{2}}}\right] a=0,
$$

which shows the evolution of the normalized vector potential in pair plasmas. Here $a=$ $e A / m c^{2}$ is the normalized vector potential, $A$ is the amplitude of the circularly polarized vector potential, $m$ is the electron mass, $e$ is the magnitude of the electron charge, $c$ is the speed of light in vacuum, $\beta=2 \beta_{e} \beta_{p} /\left(\beta_{e}+\beta_{p}\right)$ is the dimensionless temperature parameter with $\beta_{e, p}=\left(c / v_{T e, p}\right)^{2}, v_{T e, p}=\left(T_{e, p} / m\right)^{1 / 2}$ is the thermal speed, $T_{e}\left(T_{p}\right)$ is the electron (positron) temperature, we have normalized the time and co-moving spatial variables by $\omega_{0} / \omega_{\mathrm{p}}^{2}$ and $c / \omega_{\mathrm{p}}$, respectively, $\omega_{\mathrm{p}}=\left(4 \pi n_{0} e^{2} / m\right)^{1 / 2}$ is the electron plasma frequency, $n_{0}$ is the unperturbed electron density, and $\omega_{0}$ is the central wave frequency. Numerical analysis of Eq. (1) revealed that weakly modulated electromagnetic (em) pulses would undergo collapse, leading to strong intensification of the localized em pulses.

Even though Eq. (11) contains a saturation nonlinearity, halting of collapse could be obtained by spectral broadening techniques, well-known in inertial confinement fusion. Moreover, in many applications of such an equation, for example astrophysical systems, the pulses can be partially coherent. Thus, understanding the dynamics and stability of electromagnetic pulses taking incoherence effects into account may be important for practical purposes.

A canonical way for analyzing effects of partial coherence is to use the so called Wigner function, defined as the Fourier transform of the two-point correlation function of the vector potential $a$ according to [28, 29]

$$
\rho(t, \mathbf{r}, \mathbf{k})=\frac{1}{(2 \pi)^{3}} \int d \boldsymbol{\xi} e^{i \mathbf{k} \cdot \boldsymbol{\xi}}\left\langle a^{*}(t, \mathbf{r}+\boldsymbol{\xi} / 2) a(t, \mathbf{r}-\boldsymbol{\xi} / 2)\right\rangle,
$$

where the angular bracket denotes the ensemble average. The Wigner function represents a generalized distribution function for quasi-particles, in this case photons. From the definition (21), one finds the relation

$$
I(t, \mathbf{r})=\int d \mathbf{k} \rho(t, \mathbf{r}, \mathbf{k})
$$


where $I=\left\langle|a|^{2}\right\rangle$. Applying the time derivative to the definition (2) and using Eq. (11), we obtain the kinetic equation

$$
\frac{\partial \rho}{\partial t}+\mathbf{k} \cdot \nabla \rho-\frac{2 \exp [\beta(1-\sqrt{1+I})]}{\sqrt{1+I}} \sin \left(\frac{1}{2} \overleftarrow{\nabla} \cdot \vec{\nabla}_{k}\right) \rho=0
$$

for the quasi-particles. Here the sin-operator is defined in terms of its Taylor expansion. Keeping only the first term in the latter, which corresponds to the long wavelength limit, we obtain a photon kinetic (or the Liouville) equation

$$
\frac{\partial \rho}{\partial t}+\mathbf{k} \cdot \nabla \rho-\nabla\left[\frac{\exp [\beta(1-\sqrt{1+I})]}{\sqrt{1+I}}\right] \cdot \nabla_{k} \rho=0 .
$$

However, the photon kinetic limit is only valid for weak spatial variations in $\rho$, and produces a modulational instability growth rate which is unbounded as the wavenumber of the photons increases. Thus, for a broad spatial spectral distribution of photons the dynamics of the Liouville equation is highly unstable and strongly deviates from the full Wigner dynamics, and will therefore not be analyzed further here.

Equations (3) and (4) constitute a complete description of partially coherent nonlinear photons in an electron-positron plasma.

Next, we perform a perturbation analysis of Eq. (4) . Letting $\rho=\rho_{0}(\mathbf{k})+\rho_{1} \exp (i \mathbf{K}$. $\mathbf{r}-i \Omega t$ ), where $\left|\rho_{i}\right| \ll \rho_{0}$, we linearize Eqs. (3) and (4) with respect to the perturbation variables. From Eq. (4) we then obtain

$$
-i \Omega \rho_{1}+i \mathbf{k} \cdot \mathbf{K} \rho_{1}-2 I_{1} \frac{d U\left(I_{0}\right)}{d I_{0}} \sin \left(\frac{i}{2} \mathbf{K} \cdot \nabla_{k}\right) \rho_{0}=0,
$$

where

$$
U\left(I_{0}\right)=\frac{\exp \left[\beta\left(1-\sqrt{1+I_{0}}\right)\right]}{\sqrt{1+I_{0}}} .
$$

Combining (5) with Eq. (3) we obtain the nonlinear dispersion relation

$$
1=\frac{d U\left(I_{0}\right)}{d I_{0}} \int d \mathbf{k} \frac{\rho_{0}(\mathbf{k}-\mathbf{K} / 2)-\rho_{0}(\mathbf{k}+\mathbf{K} / 2)}{\Omega-\mathbf{k} \cdot \mathbf{K}},
$$

which is valid for partially coherent intense laser pulses in an electron-positron plasma.

In the case of a monochromatic pulse, the background distribution function satisfies $\rho_{0}(\mathbf{k})=I_{0} \delta\left(\mathbf{k}-\mathbf{k}_{0}\right)$ for some wavevector $\mathbf{k}_{0}$. The dispersion relation (8) then reads

$$
\Omega=\mathbf{K} \cdot \mathbf{k}_{0} \pm\left[\frac{1}{4} K^{4}+I_{0} \frac{d U\left(I_{0}\right)}{d I_{0}} K^{2}\right]^{1 / 2}
$$


which is agreement with the results found in Ref. 31]. The growth rate $\Gamma=-i \Omega$ is given by

$$
\Gamma=\left[\frac{I_{0}}{2}\left(\beta+\frac{U\left(I_{0}\right)}{1+I_{0}}\right) K^{2}-\frac{1}{4} K^{4}\right]^{1 / 2} .
$$

In order to simplify the perturbation analysis, we introduce the assumption of onedimensional partially coherent photon propagation along the $z$-axis. We investigate the case of partial coherence using the background Lorentz distribution

$$
\rho_{0}(k)=\frac{I_{0}}{\pi} \frac{\Delta}{\left(k-k_{0}\right)^{2}+\Delta^{2}},
$$

where $k=k_{z}$. The distribution (11) corresponds to a partially coherent phase of the vector potential $a_{0}$, giving rise to a spectral broadening of $\rho_{0}$ with a width $\Delta$. From the dispersion relation (8) we then obtain

$$
\Omega=-i K \Delta+K k_{0} \pm\left[\frac{1}{4} K^{4}+I_{0} \frac{d U\left(I_{0}\right)}{d I_{0}} K^{2}\right]^{1 / 2}
$$

thus giving (9) in the limit $\Delta \rightarrow 0$. We see that the effect of the spectral broadening is to reduce the growth rate according to

$$
\Gamma=-K \Delta+\left[\frac{I_{0}}{2}\left(\beta+\frac{U\left(I_{0}\right)}{1+I_{0}}\right) K^{2}-\frac{1}{4} K^{4}\right]^{1 / 2} .
$$

We note that as $\Delta \rightarrow 0$, we retrieve the expression (10).

A comparison between the coherent and incoherent modulational instability growth rates is presented in Fig. 1. We note from Eq. (13) that the growth rate is larger than zero only if $\Delta<\left(I_{0} / 2\right)^{1 / 2}\left[\beta+U\left(I_{0}\right) /\left(1+I_{0}\right)\right]^{1 / 2}$, and in the small amplitude limit $I_{0} \ll 1$ we have the condition $\Delta<\left(I_{0} \beta / 2\right)^{1 / 2}$ for the modulational instability. The Liouville equation is obtained from the Wigner's kinetic equation when the product of the spatial length scale $L$ and the spectral width $\Delta$ is large enough. For ultra-short laser pulses with a focal intensities of $\mathcal{I} \approx \omega_{0}^{2}|A|^{2} / c \sim 10^{21} \mathrm{~W} / \mathrm{cm}^{2}$, wavelength $\lambda_{0} \sim 800 \mathrm{~nm}$, and spectral width $\delta \lambda \sim 50 \mathrm{~nm}$ (relevant for e.g. the Astra laser and its upgrade Astra Gemini at the Rutherford Appleton Laboratory, Oxfordshire, U.K.), we have the coherence length $\ell_{c}=\lambda_{0}^{2} / \delta \lambda \approx 13 \mu \mathrm{m}$. Since $\Delta \sim c / \ell_{c} \omega_{p}$ we have the modulational instability criteria $\left(n_{0} / n_{\text {crit }}\right)\left(c / v_{T e}\right)^{2}>m^{2} c^{5} / 4 \pi e^{2} \mathcal{I} \ell_{c}^{2}$, where $n_{\text {crit }}=m \omega_{0}^{2} / 4 \pi e^{2}$. For the above intensity the criteria for the modulational instability to take place then becomes $2.1 \times 10^{-6}<\left(n_{0} / n_{\text {crit }}\right)\left(c / v_{T e}\right)^{2}$, and if the electron temperature is $T_{e} \sim 5 \mathrm{keV}$, we find that $n_{0}>4 \times 10^{-22} n_{\text {crit }} \sim 10^{-2} \mathrm{~cm}^{-2}$. 


\section{NONLINEAR DYNAMICS OF BROADBAND INTENSE ELECTROMAG- NETIC PULSES}

In order to understand the long term behavior of modulationally unstable broadband intense electromagnetic pulses in pair plasmas, we carry out the numerical analysis of the kinetic photon equation (4) in the one-dimensional case. Accordingly, we numerically solve the set of equations

$$
\frac{\partial \rho}{\partial t}+k_{x} \frac{\partial \rho}{\partial x}-\frac{2 \exp [\beta(1-\sqrt{1+I})]}{\sqrt{1+I}} \sin \left(\frac{1}{2} \frac{\overleftarrow{\partial}}{\partial x} \cdot \frac{\vec{\partial}}{\partial k_{x}}\right) \rho=0
$$

and

$$
I(t, x)=\int_{-\infty}^{\infty} d k_{x} \rho\left(t, x, k_{x}\right) .
$$

For the numerical solutions of Eqs. (14) and (15), we use a Fourier method for the Vlasov-Poisson system of equations [32, 33], which is slightly modified to solve the Wigner equation. For this purpose, we use the Fourier transform pair

$$
\begin{aligned}
& \rho\left(t, x, k_{x}\right)=\int_{-\infty}^{\infty} d \eta \widehat{\rho}(t, x, \eta) e^{-i \eta k_{x}}, \\
& \widehat{\rho}(t, x, \eta)=\frac{1}{2 \pi} \int_{-\infty}^{\infty} d k_{x} \rho\left(t, x, k_{x}\right) e^{i \eta k_{x}},
\end{aligned}
$$

to obtain the Fourier transformed version of Eqs. (14) and (15),

$$
\frac{\partial \widehat{\rho}}{\partial t}-i \frac{\partial^{2} \widehat{\rho}}{\partial \eta \partial x}+\widehat{\rho} \sin \left(\frac{i \eta}{2} \frac{\partial}{\partial x}\right)\left[\frac{2 \exp [\beta(1-\sqrt{1+I})]}{\sqrt{1+I}}\right]=0
$$

and

$$
I(t, x)=2 \pi \widehat{\rho}(t, x, \eta)_{\eta=0},
$$

respectively. The Fourier transformed system of Eqs. (18) and (19), which is equivalent to Eqs. (14) and (15), is solved numerically in a periodic box in $x$ space, where the $x$ derivatives are approximated with a pseudo-spectral method. In this method, the differential operator $\partial / \partial x$ turns into a multiplication by $i K_{j}$ and the operator $\sin [(i \eta / 2) \partial / \partial x]$ turns into a multiplication by $\sin \left(-\eta K_{j} / 2\right)$, where $K_{j}=2 \pi j / L$ is the spatial wavenumber, $j=$ $0, \pm 1, \pm 2 \ldots$ and $L$ is the length of the spatial domain. We note that the integral over $k_{x}$ in Eq. (15) is transformed into a simple evaluation of $\widehat{\rho}$ at $\eta=0$ in Eq. (19). As an initial condition for the simulation, we take the Fourier transformed Lorentz distribution (11)

$$
\widehat{\rho}_{0}(\eta)=\frac{I_{0}}{2 \pi} \exp \left(-\Delta|\eta|+i k_{0} \eta\right)
$$


and the same parameters as in Fig. 1. In Figs. 2 and 3, we use $I_{0}=1 / 4, \Delta=0.1$, and $k_{0}=0.1$ as initial conditions. A small amplitude noise (random numbers of order $10^{-3}$ ) is added to the initial condition to give a seed for the modulational instability.

We display the temporal evolution of the em wave intensity $I$ in Fig. 2. Here we see an initially linear growth phase and a wave collapse that takes place around $t=90$. The wavelength of the collapsing pulse is approximately $\lambda=10$, and thus the most unstable mode is $k^{*}=2 \pi / 10 \approx 0.6$, in good agreement with the theoretical prediction in Fig. 1 . After the initial pulse collapse, the solution shows a chaotic behavior, where some localized envelopes show a semi-periodic behavior, and we also see a sequence of merging and splitting of wave groups. This behavior is somewhat similar to the Fermi-Pasta-Ulam recurrence phenomenon 34], which has been observed in nonlinear optics experiments [35] and has been explained theoretically in the framework of the nonlinear Schrödinger equation [36, 37]. In Fig. 3, we show Wigner's distribution function $\rho$ at different times. (It is obtained by numerically inverse Fourier transforming $\widehat{\rho}$ to obtain the real-valued $\rho$.) We note that the distribution function shows a complex dynamics and takes both positive and negative values, in contrast to solutions of the Vlasov equation for particles that takes only positive values. In Figs. 4 and 5 , we repeated the simulation with the larger spectral width $\Delta=0.3$ in the initial conditions. We used the same intensity $I_{0}=1 / 4$, as in the first simulation, and we used $k_{0}=0.05$. For this case, the temporal evolution of the pulse intensity, as shown in Fig. 4, exhibits a slower growth rate (compared to Fig. 2 with the smaller $\Delta=0.1$ ), and nonlinearly collapsing wave envelopes have smaller intensity maxima. The snap shots of the Wigner distribution function, depicted in Fig. 5, shows the initial instability and merging of nonlinear structures corresponding to merging wave envelopes in Fig. 4. Thus, a larger spectral width $\Delta$ leads to a slower and less violent dynamics of the wave envelopes that also have larger scale sizes than for the smaller values of $\Delta$. We recall that the theoretical treatment predicts that there is a largest $\Delta$ above which the modulational instability vanishes, and we would instead have damping of waves similar as Landau damping for the Vlasov equation.

\section{SUMMARY}

In summary, we have investigated the modulational instability and the nonlinear dynam-

ics of partially coherent intense electromagnetic (em) waves in an electron-positron plasma, 
taking into account the relativistic mass increase of the pairs as well as large scale density fluctuations that are created by the relativistic ponderomotive force of em waves. The dynamics of broadband intense em waves is governed by a photon kinetic (or the Liouville) equation. The latter is analyzed to obtain a nonlinear dispersion relation, which admits the modulational instability growth rate for a Lorentzian distribution of partially coherent intense em waves. It is found that a broadband of em waves leads to a reduction of the growth rate. Furthermore, the photon kinetic equation has been numerically solved to understand the nonlinear dynamics of modulationally unstable broadband intense em pulses. Our simulation results reveal the formation of localized em wave packets that show some similarity to the Fermi-Pasta-Ulam recurrence phenomenon. For larger values of the spectral width, the system shows a dynamics on a slower timescale and on larger length scales, in agreement with the linear theory. The present results should help to understand the nonlinear propagation of broadband intense electromagnetic waves in pair plasmas that appear in inertial confinement fusion schemes as well as in pulsar magnetosphere and supernovae remnants.

This work was partially supported by the Swedish Research Council and the Deutsche Forschungsgemeinschaft. 
[1] T. Piran, Phys. Rep. 314, 575 (1999); Rev. Mod. Phys. 76, 1143 (2004).

[2] P. A. Sturrock, Astrophys. J. 164, 529 (1971).

[3] J. Arons and E. T. Scharlemann, Astrophys. J. 231, 854 (1979).

[4] F. C. Michel, Rev. Mod. Phys. 54, 1 (1982).

[5] E. M. Campbell and W. J. Hogan, Plasma Phys. Control. Fusion 41, B39 (1999).

[6] T. E. Cowan, M. D. Perry, M. H. Key et al., Laser Part. Beams 17, 773 (1999).

[7] P. Helander and D. J. Ward, Phys. Rev. Lett. 90, 135004 (2003).

[8] B. E. Blue, C. E. Clayton, C. L. O'Connell et al., Phys. Rev. Lett. 90, 214801 (2003).

[9] R. G. Greaves and C. M. Surko, Phys. Rev. Lett. 75, 3846 (1995).

[10] S. I. Popel, S. V. Vladimirov, and P. K. Shukla, Phys. Plasmas 2, 716 (1995).

[11] B. Eliasson and P. K. Shukla, Phys. Rev. E 71, 046402 (2005).

[12] B. Eliasson and P. K. Shukla, Phys. Rep. 422, 225 (2006).

[13] B. Eliasson and P. K. Shukla, Phys. Plasmas 12, 104501 (2005).

[14] P. K. Shukla, N. N. Rao, M. Y. Yu et al., Phys. Rep. 138, 1 (1986).

[15] R. Bingham, J. T. Mendonca, and P K. Shukla, Plasma Phys. Control. Fusion 46, R1 (2004).

[16] L. Stenflo, P. K. Shukla and M. Y. Yu, Astrophys. Space Sci. 117, 303 (1985).

[17] M. Y. Yu, P. K. Shukla and L. Stenflo, Astrophys. J. 309, L63 (1986).

[18] V. I. Berezhiani and S. M. Mahajan, Phys. Rev. Lett. 73, 1110 (1994).

[19] G. A. Mourou, T. Tajima, and S. V. Bulanov, Rev. Mod. Phys. 78, 309 (2006).

[20] V. V. Goloviznin and T. J. Schep, JETP Lett.70, 450 (1999).

[21] B. Shen and M. Y. Yu, Phys. Rev. Lett. 89, 275004 (2002).

[22] R. Bingham, Nature 424, 258 (2003).

[23] S. V. Bulanov, T. Esirkepov, and T. Tajima, Phys. Rev. Lett. 91, 085001 (2003).

[24] R. A. Cairns, A. Reitsma, and R. Bingham, Phys. Plasmas 11, 766 (2004).

[25] M. Marklund, and P. K. Shukla, Rev. Mod. Phys. 78, 591 (2006).

[26] C. J. McKinstrie and R. Bingham, Phys. Fluids B 4, 2626 (1992); A. S. Sakharov and V. I. Kirsanov, Phys. Rev. E 49, 3274 (1994); S. Guérin, G. Laval, P. Mora et al., Phys. Plasmas 2, 2807 (1995); B. Quesnel, P. Mora, J. C. Adam, S. Guérin et al., Phys. Rev. Lett. 78, 2132 (1997); H. C. Barr, P. Mason, and D. M. Parr, Phys. Rev. Lett. 83, 1606 (1999). 
[27] P. K. Shukla, M. Y. Yu and N. L. Tsintsadze, Phys. Plasmas 27, 327 (1984).

[28] J. T. Mendonça, Theory of Photon Acceleration (Institute of Physics Publishing, Bristol, 2001).

[29] E. P. Wigner, Phys. Rev. 40, 749 (1932).

[30] R. Fedele and D. Anderson, J. Opt. B: Quantum Semiclass. Opt. 2, 207 (2000).

[31] P. K. Shukla, M. Marklund, and B. Eliasson, Phys. Lett. A 324, 193 (2004).

[32] B. Eliasson, J. Sci. Comput. 16, 1 (2001).

[33] B. Eliasson, Comput. Phys. Commun. 170, 205 (2006).

[34] D. K. Campbell, P. Rosenau, and G. M. Zaslavsky, Chaos 15, 015101 (2005).

[35] G. Van Simaeys, Ph. Emplit, and M. Haelterman, Phys. Rev. Lett. 87, 033902 (2001).

[36] E. Infield, Phys. Rev. Lett 47, 717 (1981).

[37] N. N. Akhmediev and V. E. Korneev, Theor. Math. Phys. 69, 1089 (1986). [Teor. Mat. Fiz. 69, 195 (1986).] 
Figure 1: The modulational instability growth rates plotted as a function of $K$, as given by the expression (13). We have used $I_{0}=1 / 4$ and $\beta=1$. The thick curve represents the coherent case with $\Delta=0$, the thin curve has a finite spectral width $\Delta$ of 0.1 . The decreased growth rate due to spectral broadening can clearly be seen.

Figure 2 (Color online): The intensity $I$ as a function of $x$ and $t$. The parameters used in initial are condition $I=I_{0}=1 / 4, \Delta=0.1$ and $k_{0}=0.1$.

Figure 3 (Color inline): The Wigner distribution function $\rho$ as a function of $x$ and $k_{x}$, at times $t=0, t=85, t=100$ and $t=150$ (upper to lower panels), with the corresponding intensity in Fig. 2. For the initial conditions we used $I=I_{0}=1 / 4, \Delta=0.1$ and $k_{0}=0.1$.

Figure 4 (Color online): The intensity $I$ as a function of $x$ and $t$. The parameters used in the initial condition are $I=I_{0}=1 / 4, \Delta=0.3$ and $k_{0}=0.05$.

Figure 5 (Color online): The Wigner distribution function $\rho$ as a function of $x$ and $k_{x}$, at times $t=0, t=300, t=400$ and $t=500$ (upper to lower panels), with the corresponding intensity in Fig. 4. For the initial conditions we used $I=I_{0}=1 / 4, \Delta=0.3$ and $k_{0}=0.05$. 


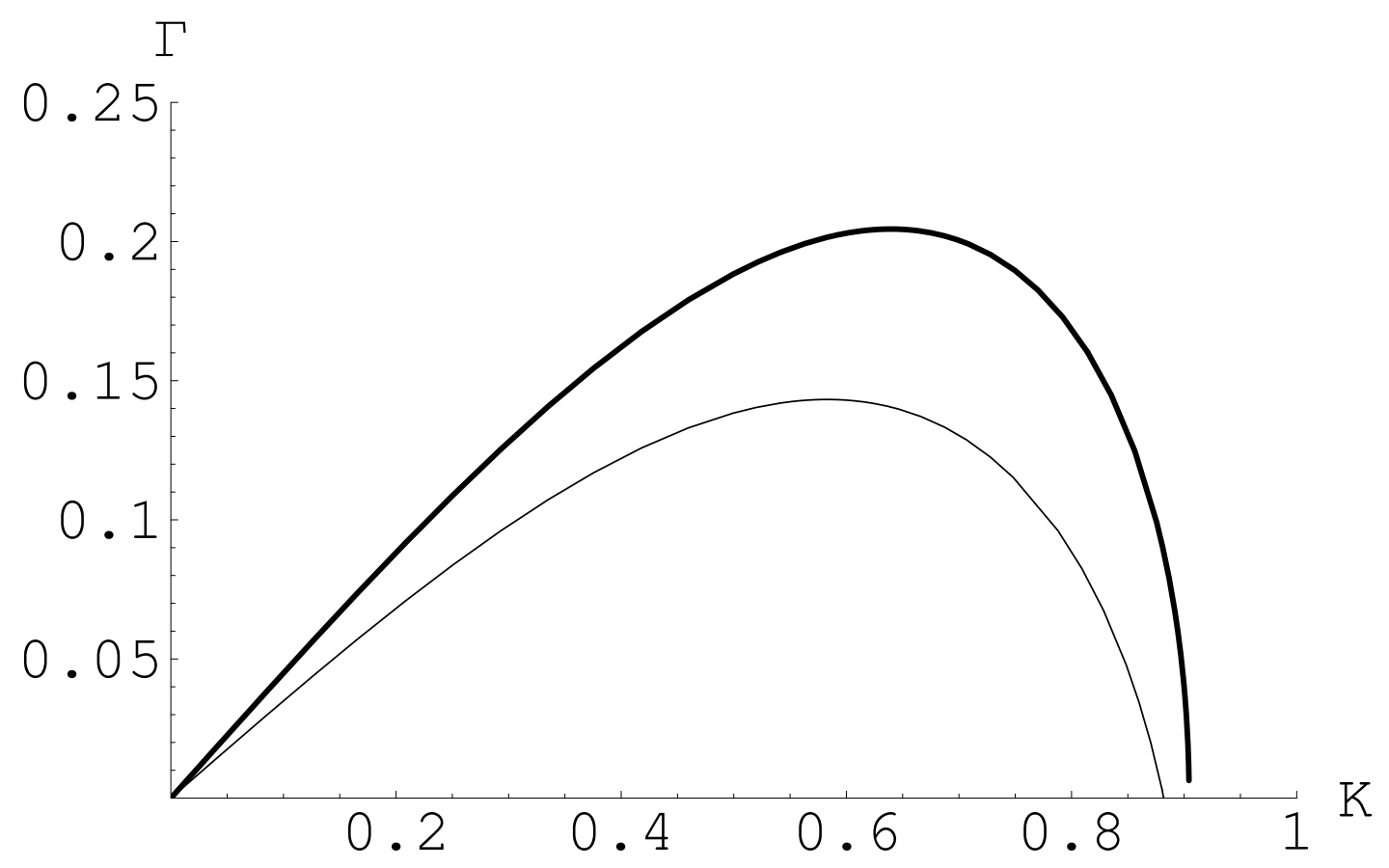

FIG. 1:

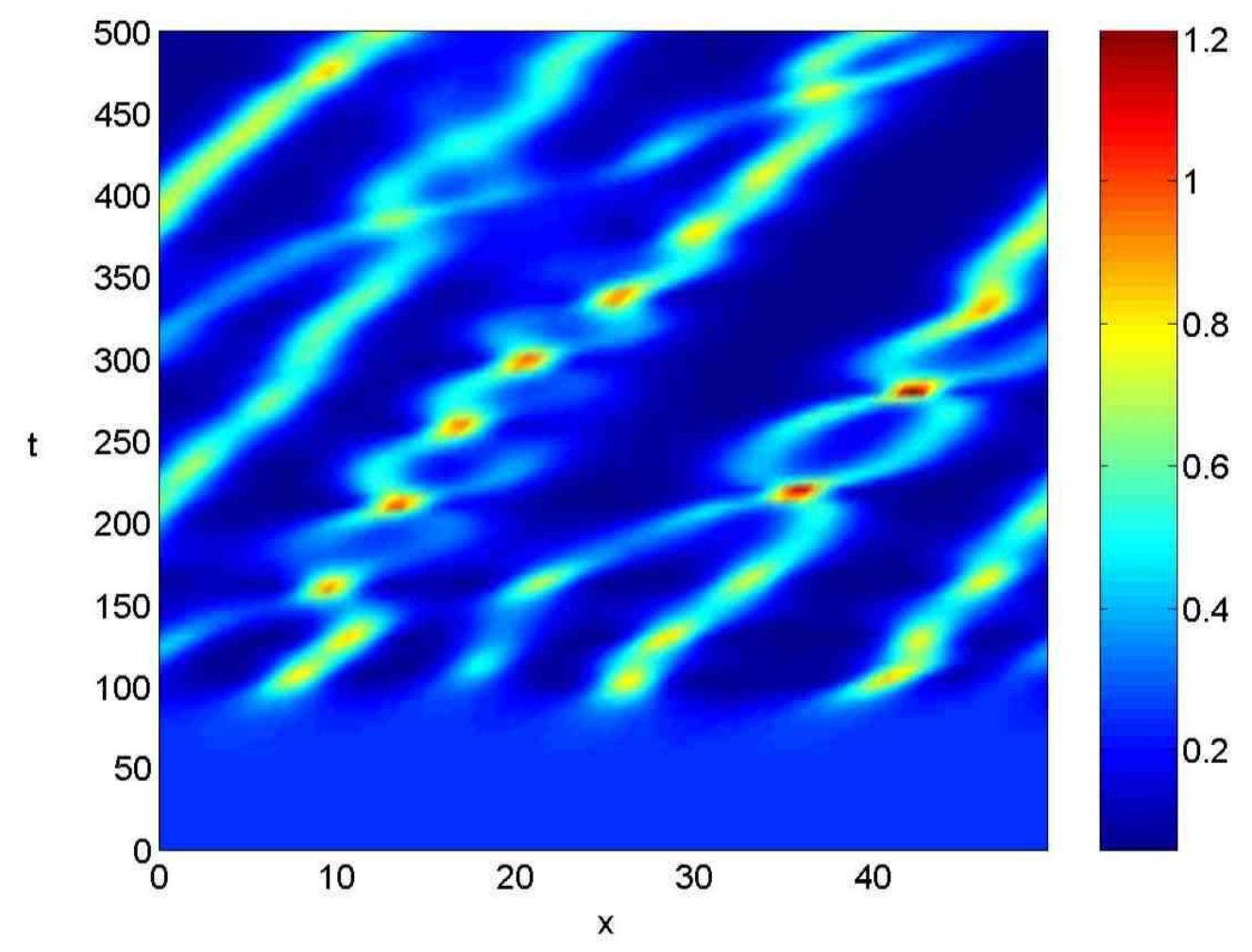

FIG. 2: 

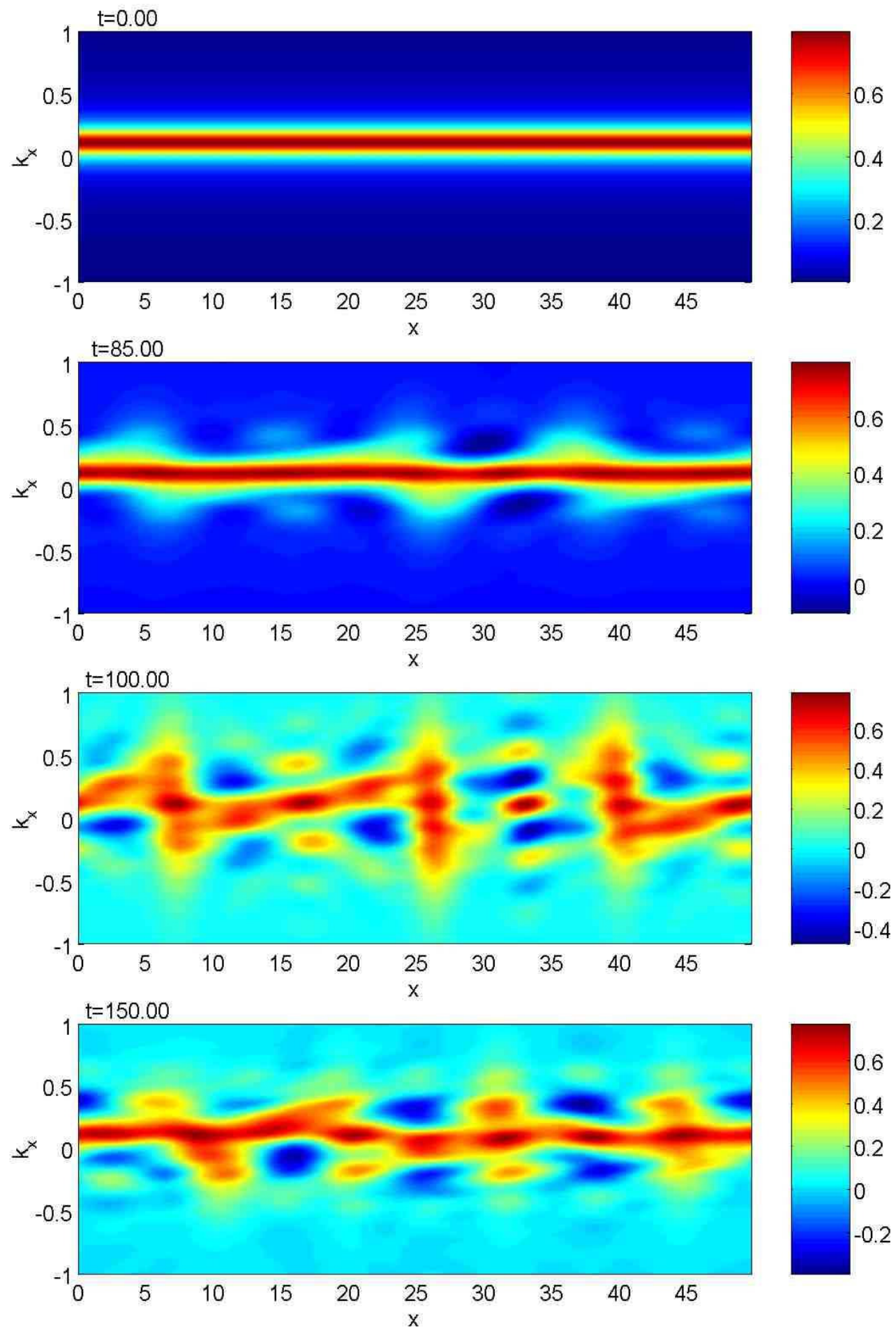

FIG. 3: 


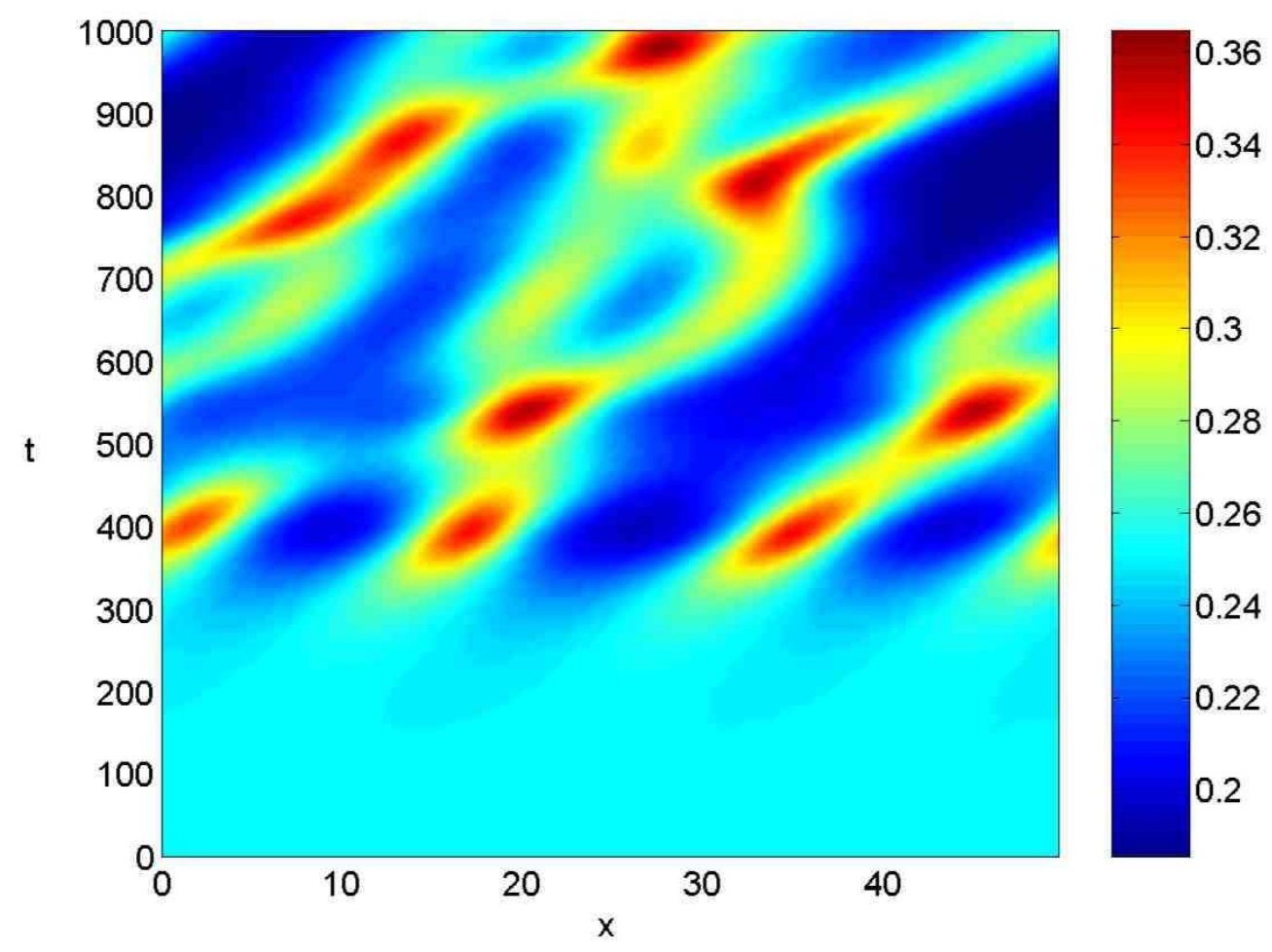

FIG. 4: 

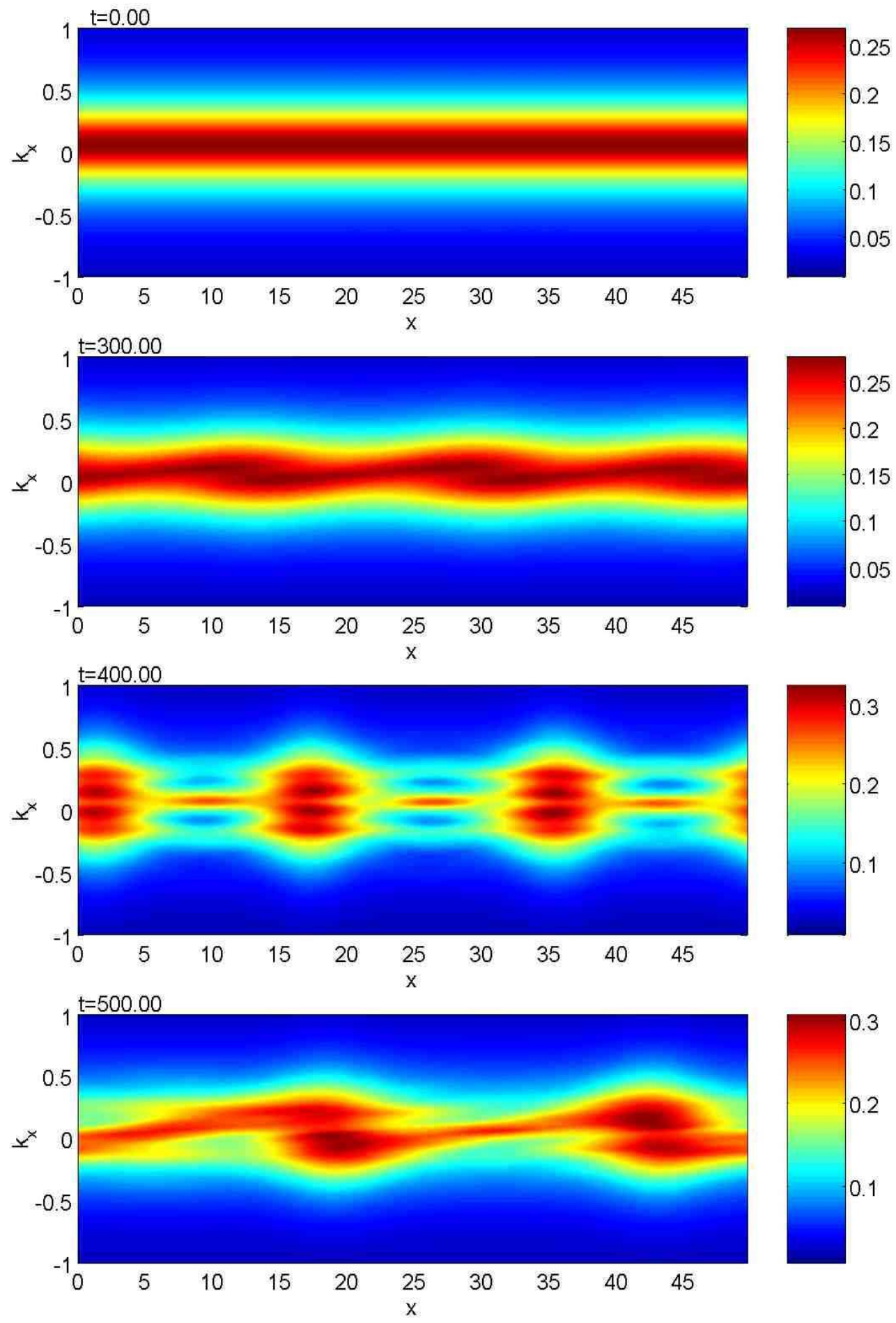

FIG. 5: 